\title{
Helicobacter pylori antibiotic sensitivity pattern in dyspeptic patients in Kano, Nigeria
}

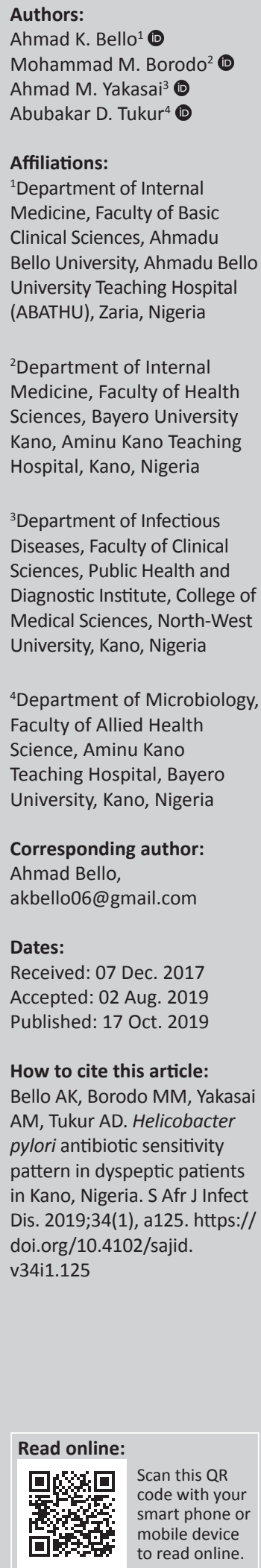

Background: Despite the high prevalence of Helicobacter pylori infection in Nigeria, in the North-West there are no studies on the antibiotic sensitivity pattern of this organism. This study aims to determine the antibiotic sensitivity pattern of this bacterium as well as bridge the gap in knowledge.

Methods: The study was cross-sectional in design. Questionnaires were administered in dyspeptic patients to obtain the relevant data. Two sets of gastric biopsy specimens were taken during upper gastrointestinal (GI) endoscopy. One set was sent to the histopathology laboratory for assessment and $H$. pylori identification, while the other set for culture was minced and plated on Columbia blood agar media (Oxoid Ltd, England) incubated at $37^{\circ} \mathrm{C}$ in an anaerobic jar containing CampyGen (Oxoid Ltd) to provide the required micro-aerobic environment. The disc diffusion method was used in determining the sensitivity pattern of isolates. Pre-treatment and post-treatment stool samples were collected from each patient for a H. pylori faecal antigen test to assess eradication rate.

Results: The sensitivity of $H$. pylori to amoxicillin was $9.2 \%$, and $100 \%$ for both clarithromycin and levofloxacin. Tetracycline, metronidazole, cefuroxime, tinidazole and ciprofloxacin were $100 \%$ resitant. The prevalence of $H$. pylori at histology was $81.7 \%$. Only 101 subjects had a positive H. pylori stool antigen test.

Conclusion: This study showed a high amoxicillin resistance; however, there is high sensitivity $(100 \%)$ to clarithromycin and levofloxacin. We recommended that levofloxacin be adopted in preference to amoxicillin as part of triple therapy in Nigeria.

Keywords: H. pylori Antibiotic Sensitivity; Kano - Nigeria; H. pylori Culture; Histology; Clarithromycin and Levofloxacin Sensitivity.

\section{Introduction}

Helicobacter pylori is the most important aetiologic risk factor of peptic ulcer disease (PUD) that infects human gastric tissue in half of the world's population. There is a high prevalence of H. pylori in resource-poor regions of the world, particularly in Africa, which has the highest burden of the infection. ${ }^{1,2,3}$ With the high prevalence of $H$. pylori in our environment, not surprisingly resistance to some of the antibiotics used in its treatment is posing a huge challenge in eradicating the pathogen, leading to high recurrence of dyspeptic symptoms in patients who have completed H. pylori eradication therapy.

In a study conducted in Jos, North-central Nigeria, in 1999, H. pylori sensitivity to amoxicillin and clarithromycin was reported as 100\% and 87.3\%, respectively. ${ }^{4}$ However, recent studies in 2010 in South-West Nigeria ${ }^{5}$ reported $34 \%$ amoxicillin sensitivity, while in Cameroon, $55.3 \%$ clarithromycin sensitivity was reported, ${ }^{6}$ In the North-West zone of Nigeria (the study area), there is no data regarding the antibiotic sensitivity pattern of $H$. pylori. This study was therefore designed to determine the in vitro sensitivity pattern of $H$. pylori obtained from dyspeptic patients undergoing diagnostic oesophago-gastro-duodenoscopy (OGD) at Aminu Kano Teaching Hospital (AKTH), Kano, Nigeria. The study is also intended to bridge the gap and to contribute to the growing interest and knowledge in $\mathrm{H}$. pylori, and to assist physicians in the choice of appropriate antibiotics for $H$. pylori eradication.

\section{Methods}

The study was carried out in AKTH, a tertiary hospital in Kano, the most populous state in Nigeria, from August 2011 to July 2013. Aminu Kano Teaching Hospital is a public hospital in the

Copyright: @ 2019 . The Authors. Licensee: AOSIS. This work is licensed under the Creative Commons Attribution License. 
North-West with a functioning endoscopy and endoscopic retrograde cholangiopancreatography facility (ERCP), and thus receives referrals from many states. The study was cross-sectional or interventional in design, and the ethical approval for the study was obtained from the research ethics committee of AKTH before the commencement of the study. Dyspeptic patients 18 years and above referred for upper gastrointestinal endoscopy, who consented to participate in the study, constituted the study population and were randomly recruited to meet the calculated sample size of 320 subjects. Any patients on antibiotics, proton pump inhibitors (PPI) or bismuth salts in the preceding 4 weeks, and those with medical conditions such as congestive cardiac failure and cardiac arrhythmias, were excluded from participating in the study. Written informed consent was sought and obtained from each patient before commencing the study and the procedure.

Patients were then booked for OGD and asked to fast overnight, for at least $8 \mathrm{~h}$ to the morning of the endoscopy. Each patient was given two sterile plastic containers to provide stool on the day of the endoscopy for pre-treatment $H$. pylori faecal antigen test, and the second stool sample collected 4 weeks post-eradication therapy to assess treatment outcome. At the endoscopy suite, the endoscopy procedure was explained to the patient, and then consent for endoscopy obtained. Thereafter, $10 \%$ xylocaine pharyngeal spray was administered to the patient's pharynx. The patient was then placed on his or her left lateral position on the endoscopy couch, and a plastic dental guard was held firmly in the patient's mouth by the assisting endoscopy nurse. A forward viewing fibre-optic Pentax FC-38 LW 2008 gastroscope was gently introduced under vision to examine the oesophagus, stomach and duodenum of the patients. During the procedure, two sets of four biopsy specimens, two each from the body of the stomach and antrum, were taken and the specimen for culture were immediately placed into screw top bottles with $1 \mathrm{~mL}$ of $0.9 \%$ sterile physiologic saline and transported within $1 \mathrm{~h}$ to the microbiology laboratory for H. pylori culture and antibiotic sensitivity. The other set fixed in $10 \%$ formaldehyde and sent to the histopathology laboratory for histologic assessment and identification of H. pylori.

The biopsy specimens for culture were minced by the attending microbiology scientist and then placed in a two plate media: the first medium being Columbia blood agar (Oxoid Ltd, Basingstoke, Hampshire, England) supplemented with 7\% laked horse blood (Oxoid Ltd), and the second was Columbia blood agar (Oxoid Ltd) supplemented with 7\% sheep blood (Oxoid Ltd). Dent's supplement (Oxoid Ltd), containing vancomycin $5.0 \mathrm{mg}$, trimethoprim lactate $2.5 \mathrm{mg}$, cefsulodin $2.5 \mathrm{mg}$ and amphotericin B $2.5 \mathrm{mg}$, was also added to the two plates. Both plates were then incubated at $37^{\circ} \mathrm{C}$ in an anaerobic jar containing CampyGen (gas generating kits) (Oxoid Ltd) to provide the micro-aerobic environment required for growth of the organism. The plates were checked daily over 3-5 days for growth. Helicobacter pylori isolates were identified by using Gram stain features, urease, catalase and oxidase activities. Identified $H$. pylori colonies were then sub-cultured on Columbia blood agar supplemented with $7 \%$ sheep blood to get a heavy growth.

\section{Antimicrobial susceptibility}

Eight antimicrobial agents were tested against each $H$. pylori isolate using the antibiotic test discs (amoxicillin, levofloxacin clarithromycin and tetracycline) (Oxoid Ltd). Other drugs are metronidazole, ciprofloxacin, cefuroxime and tinidazole. Inoculum from the subculture was made and streaked onto Brucella Agar containing 7\% sheep blood. The antibiotic strips were added and then the plates were incubated at $37{ }^{\circ} \mathrm{C}$ in a micro-aerobic environment provided by the CampyGen system (Oxoid Ltd) for another 3-4 days. The antibiotics' susceptibility $(\mathrm{S})$ and resistance $(\mathrm{R})$ were recorded for each of the antibiotics previously listed.

In the histopathology laboratory, the paraffin-embedded tissue blocks were sectioned at $4 \mu \mathrm{m}$ thickness and stained with the routine $\mathrm{H}$ and $\mathrm{E}$ stain for morphology, while modified Giemsa and Warthin-Starry stains were used for the identification of $H$. pylori. Sections found to show spiral bacteria in the mucosal layer or on the surface of the gastric epithelial cells were considered positive for $H$. pylori as assessed independently by two histopathologists.

\section{Helicobacter pylori faecal antigen test}

A stool sample was collected from each patient on the day of endoscopy to assess for $H$. pylori faecal antigen using $H$. pylori Antigen RapiCard Instatest card (Cortez Diagnostics Inc., Calabasas, CA, United States [US]). If found to be positive, amoxicillin $1 \mathrm{~g}$ bid and clarithromycin $500 \mathrm{mg}$ bid along with rabeprazole $20 \mathrm{mg}$ od were prescribed to the patients to take for 14 days, and they were instructed to bring a second stool sample 4 weeks after completing the medications (post-treatment) to assess the eradication or otherwise. The faecal antigen tests were performed on fresh stool samples according to the manufacturer's direction as described below. The required numbers of sample diluent vials were labelled with the sample identity number and the cap removed. The applicator stick, which was attached to the cap of the stool specimen bottle, was used to transfer a small piece of formed stool into the sample bottle containing specimen preparation buffer, whereas a liquid or semi-solid stool sample $(100 \mathrm{~mL}$ of sample) was added to the vial using a disposable pipette. The cap was replaced and the mixtures homogenised for 15 seconds by manually shaking vigorously. With the cassette removed from the sealed foil pouch, the sample bottle tip was snapped off and three drops of diluted sample was added to the well. The result was read within 10-15 minutes.

\section{Statistical analysis}

Data obtained were analysed using Statistical Package for Social Sciences, version 16.0 (SPSS Inc., 2009, Chicago, IL, US). Quantitative variables were summarised using 
range, means and standard deviation, while qualitative variables were summarised using ratios, proportions and percentages. A value of $p<0.05$ was regarded as statistically significant. Determinants and predictors were explored using univariate and multivariate analyses with logistic regression.

\section{Ethical considerations}

Ethical approval to conduct the research on antibiotic sensitivity pattern in dyspeptic patients in Kano, Nigeria, was obtained from the Ethical Committee of the Aminu Kano Teaching Hospital, Kano, Nigeria (clearance number: AKTH/ MAC/SUB/12A/P3/IV/608).

\section{Results}

Table 1 shows $136(44.4 \%)$ participants were males, while $170(55.6 \%)$ were females, with a male:female ratio of 1:1.3. The ages of the subjects ranged from 18 to 84 years, with a mean age of $41.2 \pm 15.3$ years.

Of the 320 samples sent to the histology laboratory for analysis, 14 (4.4\%) were lost during processing. The remaining 306 samples were examined by the pathologist, out of which $250(81.7 \%)$ were positive for $H$. pylori, while $56(18.3 \%)$ were negative. This gave H. pylori a prevalence of $81.7 \%$ at histology.

\section{Helicobacter pylori culture}

Out of the 320 samples cultured, 109 grew H. pylori, while the remaining 211 were negative, as seen in Table 2. Of the 109 subjects who were $H$. pylori culture positive, 55 (50.5\%) were males, while 54 (49.5\%) were females, with male:female ratio of $1: 1$

\section{Helicobacter pylori antibiogram}

As depicted in Table 3, the sensitivity of the 109 H. pylori isolates cultured is as follows.

TABLE 1: The prevalence of Helicobacter pylori according to gender distribution of the study subjects.

\begin{tabular}{lccc}
\hline Sex & Subjects & H. pylori positives & Prevalence rate (\%) \\
\hline Males & 136 & 112 & 82.4 \\
Females & 170 & 138 & 81.2 \\
Total & 306 & 250 & 81.7 \\
\hline
\end{tabular}

H. pylori, Helicobacter pylori.

TABLE 2: Endoscopic finding with distribution of Helicobacter pylori at culture.

\begin{tabular}{|c|c|c|c|c|c|}
\hline \multicolumn{6}{|c|}{ Helicobacter pylori culture } \\
\hline \multirow[t]{2}{*}{ Endoscopic finding } & \multicolumn{2}{|c|}{ Positive } & \multicolumn{2}{|c|}{ Negative } & \multirow[t]{2}{*}{$p$} \\
\hline & $N$ & $\%$ & $N$ & $\%$ & \\
\hline Normal & 24 & 49.0 & 25 & 51.0 & 0.56 \\
\hline Duodenal ulcer & 6 & 66.7 & 3 & 33.3 & 0.31 \\
\hline Gastric ulcer & 11 & 36.7 & 19 & 63.3 & 0.90 \\
\hline Gastric cancer & 1 & 12.5 & 7 & 87.5 & 0.43 \\
\hline Gastritis & 42 & 29.8 & 98 & 70.2 & 0.90 \\
\hline Duodenitis & 23 & 45.1 & 28 & 54.9 & 0.69 \\
\hline Oesophagitis & 2 & 8.7 & 21 & 91.3 & 0.20 \\
\hline Oesophageal cancer & 0 & 0.0 & 3 & 100 & 0.27 \\
\hline Hiatus hernia & 0 & 0.0 & 2 & 100 & 0.36 \\
\hline Candida oesophagitis & 0 & 0.0 & 4 & 100 & 0.21 \\
\hline Total & 109 & 34.1 & 211 & 65.9 & - \\
\hline
\end{tabular}

All $109(100 \%)$ H. pylori isolates were sensitive to clarithromycin and levofloxacin, but only 10 (9.2\%) isolates were sensitive to amoxicillin. None of the isolates were sensitive to metronidazole, tetracycline, tinidazole, ciprofloxacin and cefuroxime.

\section{Discussion}

The prevalence of $H$. pylori in this study was $81.7 \%$ using histology, as seen in Table 1, which is the gold standard for the detection of H. pylori in our environment (Figure 1). This prevalence is consistent with $H$. pylori prevalence rates as reported in other Nigerian studies. An earlier study in 2009 from the same centre by Bashir and $\mathrm{Ali}^{7}$ reported $81 \%$ prevalence. In another study from Gombe ${ }^{8}$ H. pylori prevalence was found to be $77.1 \%$, while Ndububa et al. ${ }^{9}$ in Ile-Ife reported prevalence rates of $73 \%$. Nigerian prevalence rates are similar to those reported in studies in South Africa ${ }^{10}$ and Kenya ${ }^{11}$ which reported, respectively, rates of $66 \%$ and $94 \%$. Woodward et al. ${ }^{12}$ in a study in Glasgow, United Kingdom, reported a prevalence of $66 \%$ which they noted was more typical of prevalence in developing countries and thus concluded that the high degree of social deprivation in Glasgow at that time was the explanation for the high prevalence of $H$. pylori found in the study. In our study, as shown in Table 1, the prevalence of $H$. pylori infection is higher in males $(82.4 \%)$ than females $(81.6 \%)$. Univariate analysis on demographic factors in this study showed that there is an increased prevalence of $H$. pylori in male subjects,

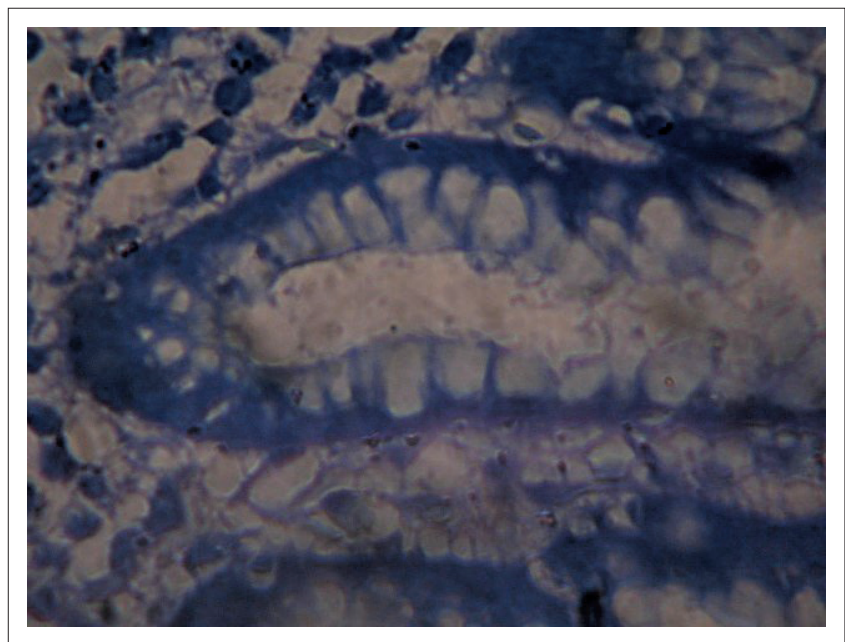

FIGURE 1: Helicobacter pylori in gastric biopsy at histology.

TABLE 3: Helicobacter pylori antibiogram.

\begin{tabular}{lcccccc}
\hline Antibiotic tested & \multicolumn{2}{c}{ Susceptible } & & \multicolumn{2}{c}{ Resistant } \\
\cline { 2 - 3 } \cline { 5 - 6 } & $\boldsymbol{N}$ & $\mathbf{\%}$ & & $\boldsymbol{N}$ & $\mathbf{\%}$ \\
\hline Amoxicillin & 10 & 9.2 & & 99 & 90.8 \\
Metronidazole & 0 & 0.0 & & 109 & 100.0 \\
Tetracycline & 0 & 0.0 & & 109 & 100.0 \\
Tinidazole & 0 & 0.0 & & 109 & 100.0 \\
Ciprofloxacin & 0 & 0.0 & & 109 & 100.0 \\
Clarithromycin & 109 & 100.0 & & 0 & 0.0 \\
Cefuroxime & 0 & 0.0 & & 109 & 100.0 \\
Levofloxacin & 109 & 100.0 & & 0 & 0.0 \\
\hline
\end{tabular}


which is statistically significant $(p<0.0041)$ when compared with that of females. Similarly, Omosor et al. ${ }^{13}$ revealed that the prevalence of $H$. pylori infection was also higher in males $(55 \%)$ than females $(51.4 \%)$. Woodward et al. also reported a higher prevalence of $H$. pylori in men than in women. ${ }^{12}$ Ford and Axon observed that male gender is a risk factor for $H$. pylori infection. ${ }^{14}$ The distribution of $H$. pylori at histology in the various endoscopic findings can be seen in Table 4.

\section{Helicobacter pylori antibiotic profile}

This study revealed an antimicrobial susceptibility rate of only $9.2 \%$ for amoxicillin, an antibiotic that is commonly used in triple therapy for H. pylori eradication in Nigeria (Table 3). Jaka et al. in a systematic review and metaanalysis involving 26 articles reported a $72.6 \% \mathrm{H}$. pylori resistance to amoxicillin, ${ }^{15}$ and concluded that prevalence of metronidazole, clarithromycin and amoxicillin resistance is high in the developing world including Africa, which, they said, could impair the first-line triple therapy of $H$. pylori infection. An earlier study in Western Nigeria ${ }^{5}$ reported 34\% amoxicillin sensitivity, while in 2007 Aboderin et al. reported $100 \%$ amoxicillin resistance in their $H$. pylori isolates. ${ }^{5}$ Ndip et al. in Cameroon reported $14.4 \%{ }^{6}$ and $\mathrm{Wu}$ et al. in a study in China ${ }^{16}$ reported a rate of $28.1 \%$. However, Ani et al. in North-Central Nigeria, ${ }^{4}$ almost two decades earlier, had reported $100 \%$ susceptibility to amoxicillin. This study coming from same part of the country highlighted the likely acquisition of resistance overtime. Other studies from Malaysia, Saudi Arabia and Lebanon reported 100\%, 99\% and $100 \%$ sensitivity to amoxicillin, respectively. ${ }^{17,18,19}$ These differences in antibiotic susceptibility in the different countries could be attributed to the differences in local antibiotic prescription practices and usage in the various countries at the time of the studies. In Nigeria, drug regulations are lacking and the few laws enacted are not enforced, thus antibiotics and other drugs are sold over the counter leading to their abuse, thus encouraging the development of resistance in the individuals later. In addition, there is widespread proliferation of cheap and substandard drugs including antibiotics in the country which encourages the development of resistance not only to H. pylori but to other infections as well. Studies have shown

TABLE 4: Endoscopic finding with distribution of Helicobacter pylori at histology.

\begin{tabular}{|c|c|c|c|c|c|c|c|}
\hline \multicolumn{8}{|c|}{ Helicobacter pylori histology } \\
\hline \multirow[t]{2}{*}{ Endoscopic finding } & \multicolumn{2}{|c|}{ Positive } & \multicolumn{2}{|c|}{ Negative } & \multicolumn{2}{|c|}{ Total } & \multirow[t]{2}{*}{$P$} \\
\hline & $N$ & $\%$ & $N$ & $\%$ & $N$ & $\%$ & \\
\hline Normal & 41 & 85.4 & 7 & 14.6 & 48 & 15.7 & 0.85 \\
\hline Duodenal ulcer & 8 & 88.9 & 1 & 11.1 & 9 & 2.9 & 0.69 \\
\hline Gastric ulcer & 25 & 83.3 & 5 & 16.7 & 30 & 9.8 & 0.97 \\
\hline Gastric cancer & 2 & 40.0 & 3 & 60.0 & 5 & 1.6 & 0.00 \\
\hline Gastritis & 121 & 89.6 & 14 & 11.0 & 135 & 44.2 & 0.03 \\
\hline Oesophagitis & 18 & 78.3 & 5 & 21.7 & 23 & 7.5 & 0.58 \\
\hline Duodenitis & 42 & 85.7 & 7 & 14.3 & 49 & 16.0 & 0.00 \\
\hline Hiatus hernia & 0 & 0 & 1 & 100.0 & 1 & 0.3 & 0.60 \\
\hline Oesophageal cancer & 0 & 0 & 3 & 100.0 & 3 & 1.0 & 0.64 \\
\hline Candida oesophagitis & 1 & 33.3 & 2 & 66.7 & 3 & 1.0 & 0.86 \\
\hline Total & 258 & 84.3 & 48 & 15.7 & 306 & 100 & - \\
\hline
\end{tabular}

that in Africa amoxicillin and ampicillin are the most abused antibiotics in both rural and urban areas because they are cheaply available in oral formulations. ${ }^{20}$

We recorded $100 \%$ sensitivity to clarithromycin (Figure 2) in this study, which was similar to that of Kimanga et al. ${ }^{11}$ in Kenya, Rizwan et al. in Saudi ${ }^{18}$ and Sharara et al. ${ }^{19}$ in Beirut, who reported 100\%, 96\% and 96\% clarithromycin susceptibility, respectively. Clarithromycin resistance the world over was reported as low, ranging from $0 \%$ to $45 \% .{ }^{18,21,22}$ Studies from Malaysia ${ }^{17}$ reported $97.9 \%$ and in the United States ${ }^{23,24} 93.9 \%$ sensitivity. However, Kumala et al. ${ }^{25}$ in Indonesia and another study from Cameroon ${ }^{6}$ reported lower rates of clarithromycin susceptibility of $72.2 \%$ and $55.3 \%$. A major contrast to the findings in our study and the others noted above is a study in Western Nigeria, ${ }^{5}$ which reported that $100 \%$ of their $H$. pylori isolates were resistant to clarithromycin. We are not aware of any other study globally that has reported 100\% clarithromycin resistance; therefore, the reason for this marked difference is not clear because clarithromycin resistance is very low and as such it is used widely in the treatment of $H$. pylori infections in most parts of the world.

All 109 (100\%) isolates in our study were susceptible to levofloxacin (Figure 2). To our knowledge, there is no study that has tested levofloxacin sensitivity in Nigeria. Norazah et al. ${ }^{17}$ in Malaysia reported $99 \%$ sensitivity to levofloxacin, while a systemic review ${ }^{26}$ showed a susceptibility rate of 88.4\% in Asia, 75.9\% in Europe and 0\% in Africa. Levofloxacin can therefore be a suitable substitute to amoxicillin in the triple therapy used for $H$. pylori eradication in Nigeria.

We found $100 \%$ resistance of our isolates to ciprofloxacin as seen in Figure 3; however, a study from Ile-Ife ${ }^{5}$ in Western Nigeria, a decade earlier, showed $15.6 \%$ ciprofloxacin resistance, and the resistance rate in Jakarta was $6.9 \% .^{25}$

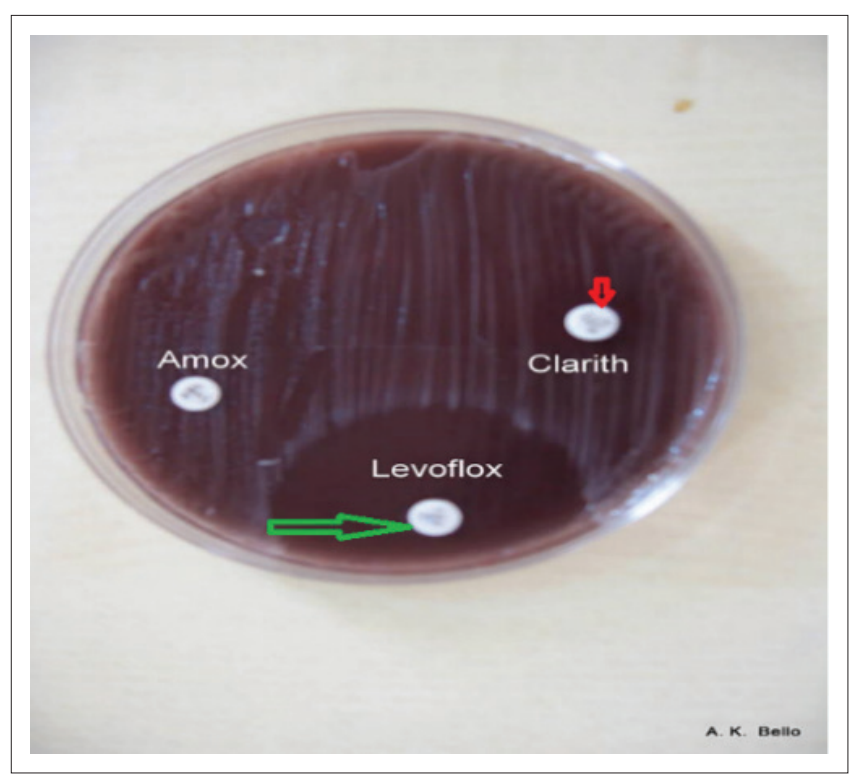

FIGURE 2: Helicobacter pylori growth inhibited by levofloxacin (green) and clarithromycin (red) above. 
The reason for this difference is not apparent; however, different antibiotic usage and abuse in the two regions as well as the time of the studies could be responsible for this difference. In Nigeria, there is tremendous pressure on the use of ciprofloxacin by general practitioners for the treatment of typhoid fever, the diagnosis that is often erroneous. Moreover, ciprofloxacin is cheap and readily available in both rural and urban areas, and thus abused by individuals and general practitioners. On the contrary, levofloxacin is new and expensive and as such not subjected to abuse, which may explain the noted susceptibility seen in our study. To our knowledge, there is no other $H$. pylori study on levofloxacin in Nigeria.

The high resistance rate of $100 \%$ observed for metronidazole and tinidazole (Figure 3) is comparable to numerous studies from many developing countries that have reported similar results to our findings. Two separate studies done in Western Nigeria reported $95 \%$ and $100 \%$ resistance to metronidazole. ${ }^{5,11}$ Studies in Cameroon, Indonesia and China reported 93.2\%, $63.9 \%$ and $100 \%$ resistance to metronidazole, respectively. ${ }^{6,25,27}$ These findings could be attributed to the poor drug regulation in Africa and other developing countries leading to high antibiotic abuse that encourages development of resistance. Metronidazole resistance has been reported in $10 \%-50 \%$ of patients in developed countries, ${ }^{28}$ whereas in developing countries virtually all strains of $H$. pylori have been found to be resistant to metronidazole. ${ }^{29}$ The high prevalence of metronidazole resistance in developing countries like Nigeria may also be related to the frequent use of imidazole derivatives for the treatment of gastrointestinal and gynaecological infections often at substandard doses for the relief of diarrhoeal disease which are of frequent occurrence. Osato et al. ${ }^{30}$ reported that metronidazole resistance was seen more in females $(63 \%)$ than their male $(35 \%)$ counterparts. From other studies, metronidazole resistance of $H$. pylori has been shown to be because of the mutational inactivation of the nitroreductase gene (rdxA gene), which encodes nitroreductase, an enzyme gene, which encodes nitro-reductase, an enzyme that reduces metronidazole to an active form. ${ }^{29}$

There was $100 \% \mathrm{H}$. pylori resistance to tetracycline in this study as seen in Figure 3, which was similar to two other

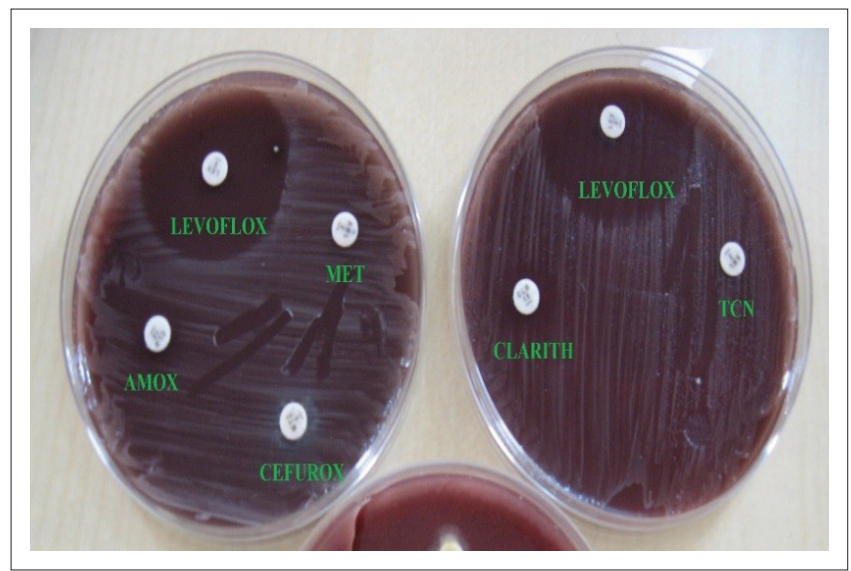

FIGURE 3: Helicobacter pylori growth un-inhibited by tetracycline, metronidazole or cefuroxime. studies by Oladiipo et al., and Smith et al. in Western Nigeria ${ }^{5,31}$ which all reported $100 \%$ tetracycline resistance. Tetracycline is not currently used for $H$. pylori eradication in Nigeria because of its high resistance profile. However, in China and Malaysia ${ }^{16,17}$ studies reported resistance rates of $1.2 \%$ and $0 \%$ to tetracycline, and Sharara in Beirut ${ }^{19}$ also reported $2 \%$ tetracycline resistance.

In this study, $100 \%$ of $H$. pylori isolates were resistant to cefuroxime (Figure 3); in contrast, a study in China ${ }^{27}$ reported resistance of only $1.1 \%$. Differences in the antibiotic usage and abuse in the two continents may be responsible for this contrast.

\section{Rapid Helicobacter pylori faecal antigen test}

One hundred and one subjects $(34.0 \%)$ were H. pylori faecal antigen test positive pre-treatment, as shown in Table 5 and Figure 4. Of the 101 initially positive, 9 patients defaulted, while 92 came back for a post-treatment eradication test (rapid H. pylori stool antigen test [Hp SAT]). Of these, $12(13.0 \%)$ were still positive post-treatment (see Figure 5 and Table 6 ). Smith et al. ${ }^{31}$ reported that $53 \%$ of their subjects

TABLE 5: Pre-treatment Helicobacter pylori faecal antigen test.

\begin{tabular}{lcc}
\hline Hp stool antigen & \multicolumn{2}{c}{ Helicobacter pylori faecal antigen test } \\
\cline { 2 - 3 } & $\boldsymbol{n}$ & $\boldsymbol{\%}$ \\
\hline Positive & 101 & 34.0 \\
Negative & 196 & 66.0 \\
\hline Total & $\mathbf{2 9 7}$ & $\mathbf{1 0 0 . 0}$ \\
\hline
\end{tabular}

Hp, Helicobacter pylori.

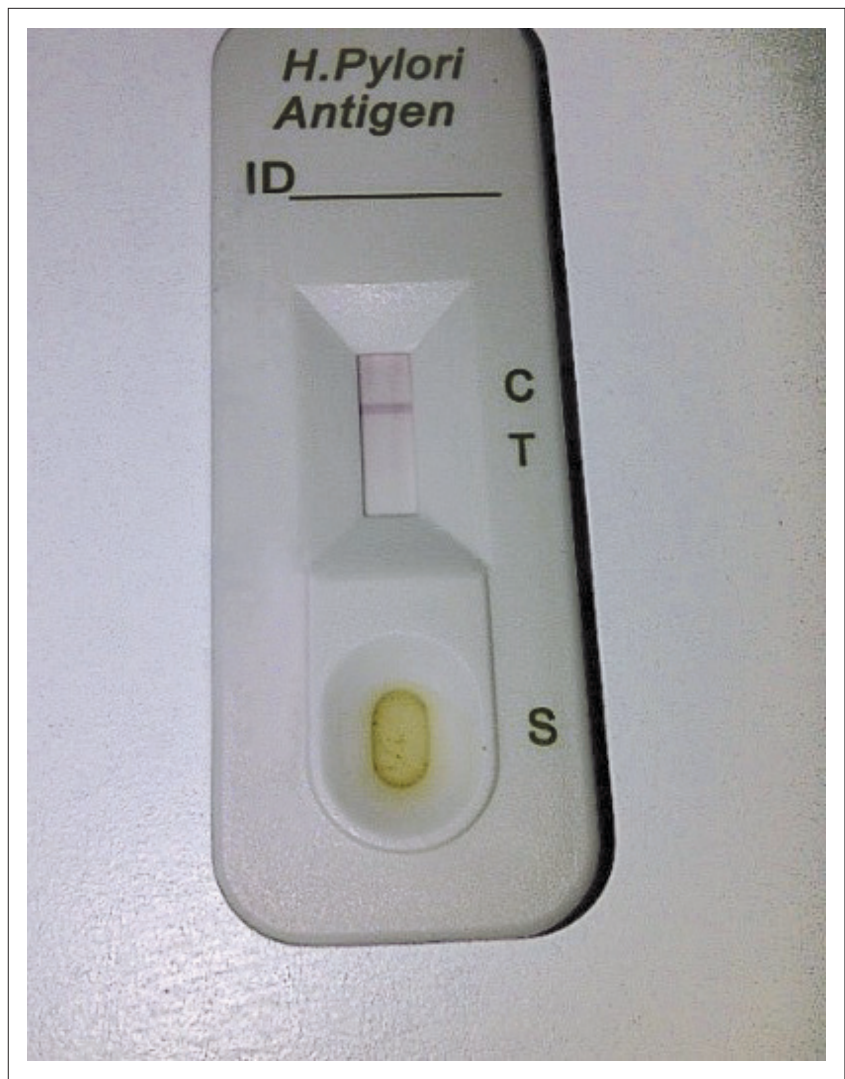

FIGURE 4: Positive Helicobacter pylori faecal antigen test (presence of horizontal line on C only). 


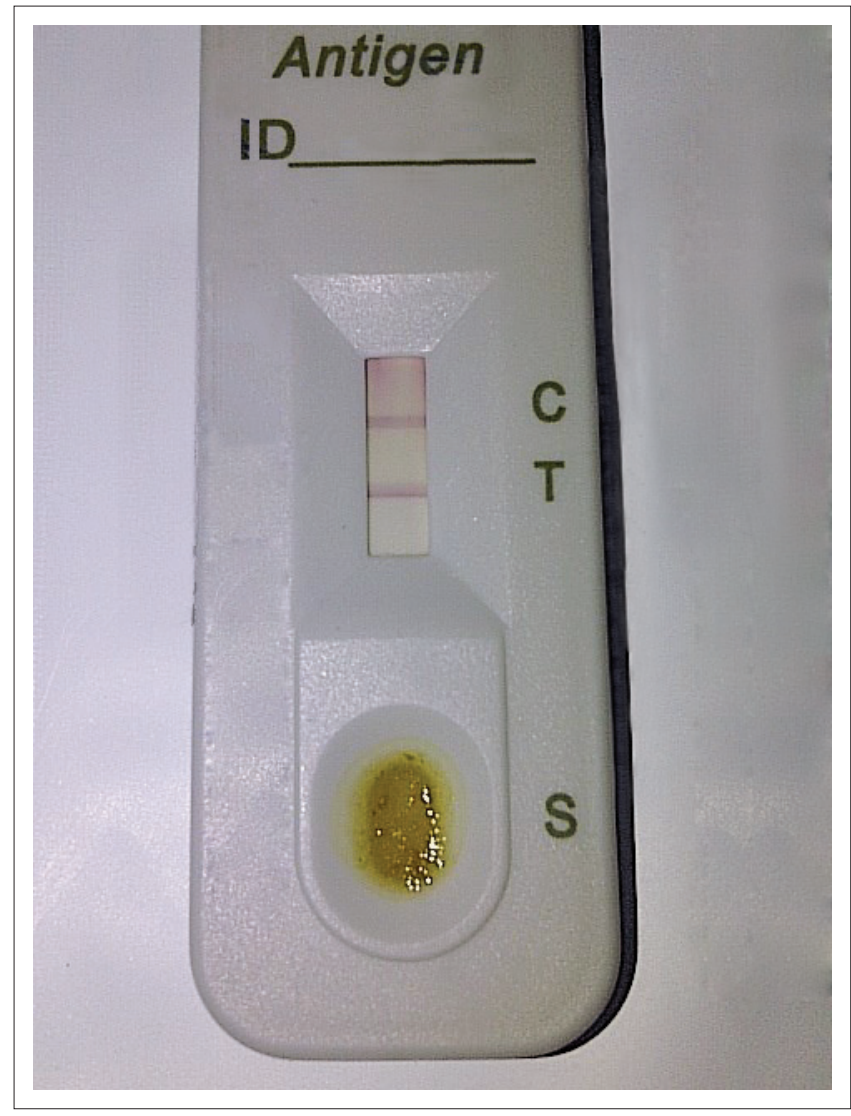

FIGURE 5: Positive Helicobacter pylori faecal antigen test (presence of horizontal lines on C \& T) above.

TABLE 6: Post-treatment Helicobacter pylori faecal antigen test.

\begin{tabular}{lcc}
\hline Hp stool antigen & \multicolumn{2}{c}{ Helicobacter pylori faecal antigen test } \\
\cline { 2 - 3 } & $\boldsymbol{n}$ & $\mathbf{\%}$ \\
\hline Positive & 12 & 13.0 \\
Negative & 80 & 87.0 \\
\hline Total & $\mathbf{9 2 \dagger}$ & $\mathbf{1 0 0 . 0}$ \\
\hline
\end{tabular}

$\mathrm{Hp}$, Helicobacter pylori.

$\dagger$, Nine patients out of the 101 positives were lost to follow-up during the post-treatment faecal antigen test.

were still positive post-treatment. The prevalence of $H$. pylori using the Hp SAT in this study was $31.6 \%$, which is similar to the $36.7 \%$ reported by Stella et al. in Lagos-Nigeria. ${ }^{32}$ Currently available eradication regimens for $H$. pylori are triple drug combination regimens comprising a PPI and two antibiotic drugs, and eradication rates of $70 \%-90 \%$ are obtained using these regimens. ${ }^{21}$ The eradication rate of $86.9 \%$ achieved by our study agrees with the above finding. The eradication rates of $H$. pylori have been lower than $80 \%$ as a result of increased resistance to $H$. pylori. ${ }^{33}$ Gisbert et al. in a systematic review of 89 studies evaluated the H. pylori stool antigen test and reported a sensitivity of $96 \%$ with a specificity of $97 \% .^{34}$ The eradication rate may have been lower if reendoscopy with $H$. pylori culture and histology was done. We probably achieved this high eradication level despite over $90 \%$ amoxicillin resistance because of the fact that proton pumps inhibitors (PPI) in themselves inhibit H. pylori. This property of PPI and the $100 \%$ susceptibility to clarithromycin of our isolates would possibly explain this high eradication rate we recorded in spite of the amoxicillin resistance. It
TABLE 7: Helicobacter pylori positivity using the three deferent methods.

\begin{tabular}{lccccc}
\hline Endoscopy findings & $\boldsymbol{n}$ & Histology & Culture & FAT 1 & FAT 2 \\
\hline Normal & 49 & 41 & 24 & 8 & 1 \\
Duodenal ulcer & 9 & 8 & 6 & 7 & 0 \\
Gastric ulcer & 30 & 25 & 11 & 16 & 2 \\
Gastric tumour & 8 & 2 & 1 & 0 & 0 \\
Gastritis & 141 & 121 & 42 & 51 & 8 \\
Duodenitis & 51 & 42 & 23 & 18 & 1 \\
Oesophagitis & 23 & 18 & 2 & 1 & 0 \\
Oesophageal tumour & 3 & 0 & 0 & 0 & 0 \\
Hiatus hernia & 2 & 0 & 0 & 0 & 0 \\
Oesophageal candida & 4 & 0 & 0 & 0 & 0 \\
\hline Total & $\mathbf{3 2 0}$ & $\mathbf{2 5 7}$ & $\mathbf{1 0 9}$ & $\mathbf{1 0 1}$ & $\mathbf{1 2}$ \\
\hline
\end{tabular}

FAT 1, pre-treatment faecal antigen test positivity; FAT 2, post-treatment faecal antigen tes positivity.

could also be explained that the free drugs (PPI, amoxicillin and clarithromycin) provided by the researcher for the required duration of treatment have encouraged patients' compliance and thus led to the achieved high eradication rate noted. The apparent high eradication rate mainly from clarithromycin effect without the support of a second antibiotic should not be taken for granted - as guidelines generally recommend - for this may expose this robust antibiotic (clarithromycin) in the future to the development of resistance by the $H$. pylori bacteria.

\section{Study limitations}

The high cost of endoscopy and the discomfort of the procedure prevented patients from agreeing to return for repeat endoscopy and biopsy (only 10 out of 320 patients agreed to return for post-treatment repeat endoscopy). We thus had to resort to in vitro testing using the H. pylori faecal antigen test which correlated well with the culture result (see Table 7) in place of the initially intended in vivo testing of the antibiotic sensitivity using a culture to determine the eradication rate.

\section{Conclusion}

This study showed that H. pylori isolates in Kano, Nigeria, are resistant to metronidazole or tinidazole, tetracycline and amoxicillin, which are among the antibiotics used in $H$. pylori eradication therapy in the country, but are sensitive to clarithromycin and levofloxacin. Therefore, this finding, though limited to only one region of the country, may provide part of the basis for the need to review the current $H$. pylori antibiotic combination therapy currently in use in Nigeria.

\section{Acknowledgement}

The authors would like to thank Prof. Sharara of the American University in Beirut for his invaluable advice, as well as Muhammad Dakata, Shaibu Kansula, Sister Kate and Sister Aishah Aliyu for their assistance in logistics while conducting this research study.

\section{Competing interests}

The authors have declared that no competing interests exist. 


\section{Author contributions}

A.K.B. initiated the study, performed the endoscopy and wrote the article. A.D.T. performed the Helicobacter pylori culture and sensitivity. A.M.Y. analysed the data. M.M.B. supervised the work and reviewed the article.

\section{Funding information}

This research received no specific grant from any funding agency in the public, commercial or not-for-profit sectors.

\section{Data availability statement}

Data sharing is not applicable to this article as no new data were created or analysed in this study.

\section{Disclaimer}

The views and opinions expressed in this article are those of the authors and do not necessarily reflect the official policy or position of any affiliated agency of the authors.

\section{References}

1. Marshall BJ, Armstrong JA, McGechie DB, Glancy RJ. Attempt to fulfill Koch's postulate for pyloric Campylobacter. Med J Austr. 1985;142(8):436-439.

2. Warren JR, Marshall BJ. Unidentified curved bacilli on gastric epithelium in active chronic gastritis. Lancet. 1983;1:8336:1273-1275. https://doi.org/10.1016/S01406736(83)92719-8

3. International Agency for Research on Cancer. IARC monographs on the evaluation of carcinogenic risks to human, schistosomes, liver flukes and Helicobacter pylori. Int Agency Res Cancer. 1994;61:3-12.

4. Ani AE, Malu AO, Onah JA, Queiroz DMM, Kirschner G. Antimicrobial susceptibility test of Helicobacter pylori isolated from Jos, Nigeria. Trans R Soc Trop Med Hyg. 1999;93(6):659-661. https://doi.org/10.1016/S0035-9203(99)90089-5

5. Oladiipo AA, Abdul RA, Babatunde WO, et al. Antibiotic resitance of Helicobacter pylori from patients in lle-Ife, South-West, Nigeria. Afr Health Sci. 2007;7(3):143-147.

6. Ndip RN, Malange Takang AE, Ojongokpoko JE, et al. Helicobacter pylori isolates recovered from gastric biopsies of patients with gastro-duodenal pathologies in
Cameroon: Current status of Antibiogram. Trop Med Intern Health. 2008;13(6):848-885. https://doi.org/10.1111/j.1365-3156.2008.02062.x

7. Bashir MT, Ali BU. Peptic ulcer disease and Helicobacter pylori infection at Kano, Nigeria. Internet J Gastroenterol. 2009;8(1):1-3. https://doi.org/10.5580/21fb

8. Mustapha S, Pindiga U, Yusuph H, Goni B, Jibrin YH. Helicobacter pylori infection among dyspeptic patients at a tertiary hospital in Northern Nigeria. Int J Infect Dis. 2011;9(2):1528-1536. https://doi.org/10.5580/2694

9. Ndububa DA, Agbakwuru AE, Adebayo RA, et al. Upper gastrointestinal findings and incidence of Helicobacter pylori infection among Nigerian patients with Dyspepsia. West Afr J Med. 2001;20(2):140-5.

10. Kidd M, Louw JA, Marks IN. Helicobacter pylori in Africa: Observations on an 'enigma within an enigma'. J Gastroenterol Hepatol. 1999;14(9):851-858. https:// doi.org/10.1046/j.1440-1746.1999.01975.x

11. Kimang'a AN, Revathi G, Kariuki S, Sayed S, Devani S. Helicobacter pylori: Prevalence and antibiotic susceptibility among Kenyans. S Afr Med J. 2010;100(1):53-57.

12. Woodward M, Morrison C, McColl K. An investigation into factors associated with Helicobacter pylori infection. J Clin Epidemiol. 2000;53(2):175-181. https://doi. org/10.1016/S0895-4356(99)00171-7

13.Omosor $\mathrm{KI}$, Omasan $\mathrm{OH}$, Ibe $\mathrm{IN}$, et al. Seroprevalence of Helicobacter pylori infection and risk factors among asymptomatic subjects in Delta state, Nigeria. Adv Microbiol. 2017;7(9):641-652. https://doi.org/10.4236/aim.2017.79050
14. Ford AC, Axon AT. Epidemiology of Helicobacter pylori infection and public health implications. Helicobacter. 2010:15 Suppl 1:1-6. https://doi.org/10.1111/j.15235378.2010.00779.x

15. Jaka $H$, Rhee JA, Ostlundh $L$, et al. The magnitude of antibiotic resistance to Helicobacter pylori in Africa and identified mutations which confer resistance to antibiotics: Systematic review and meta-analysis. BMC infect Dis. 2018:18(1):193. https://doi.org/10.1186/s12879-018-3099-4

16. Wu H, Shi XD, Wang HT, Liu JX. Resistance of Helicobacter pylori to metronidazole, tetracycline and amoxycillin. J Antimicrobial Chemother. 2000;46(1):121-123. https://doi.org/10.1093/jac/46.1.121

17. Norazah A, Zakaria WR, Mohamed R. Analysis of antibiotic susceptibility patterns of Helicobacter pylori isolates from Malaysia. Helicobacter. 2010;16(1):47-51. https://doi.org/10.1111/j.1523-5378.2010.00816.x

18. Rizwan M, Fatima N, Ayesha A. Epidemiology and Pattern of Antibiotic Resistance in Helicobacter pylori: Scenario from Saudi Arabia. Saudi J Gastroenterol. 2014;20(4):212-218. https://doi.org/10.4103/1319-3767.136935

19. Sharara A, Marwan C, George FA, Kassem AB, Fadi HM. Prevalence of Helicobacter pylori resistance to metronidazole, clarithromycin, amoxycillin and tetracycline in Lebanon. Intern J Antimicrobial Agents. 2002;19(2):155-158. https://doi. org/10.1016/S0924-8579(01)00482-4

20. Gebeyetu E, Bantie L, Azage M. Inappropraite use of antibiotics and its associated factors among urban and rural communities of Bahir Dar city administration North-West Ethiopia. PLoS One. 2015;10(9):e0138179. https://doi.org/10.1371/ journal.pone.0138179

21. Adamek RJ, Suerbaum S, Pfaffenbach B, Opfenkuch W. Primary acquired Helicobacter pylori resistance to clarithromycin, metronidazole and amoxicillin influence on treatment outcome. Am J Gastroenterol. 1998;93(3):386-389. https://doi.org/10.1111/j.1572-0241.1998.00386.x

22. Everhart JE, Kruszon-Moran D, Perez-Perez GI, Tralka TS, McQuillan G. Seroprevalence and ethnic differences in Helicobacter pylori infection among adults in the United States. J Infect Dis. 2000;181(4):1359-1363. https://doi.org/ adults in the Unit

23. Megraud F. Basis for the management of drug-resistant Helicobacter pylor infection. Drugs. 2004;64(17):1893-1904. https://doi.org/10.2165/00003495200464170-00003

24. Nicoline FT, Ndip RN. A South African perspective on Helicobacter pylori: Prevalence, epidemiology and antimicrobial chemotherapy. Afr J Microbiol Res. 2013;7(21):2430-2437. https://doi.org/10.5897/AJMR2013.5594

25. Kumala W, Aziz R. Patterns of Helicobacter pylori isolate resistance to fluoroquinolones, amoxicillin, and metronidazoles. South-East Asian J Trop Med Public Health. 2006;37(5):1260.

26. De Francesco V, Giorgio F, Hassan C, et al. Worldwide H. pylori antibiotic resistance: A systematic review. J Gastrointestin Liver Dis. 2010;19(4):409-414.

27. Bai P, Zhou LY, Xiao XM, Luo Y, Ding Y. Antibiotic susceptibility profile of Helicobacter pylori isolated from Chinese patients. J Dig Dis. 2015;16(8):2980. https://doi. org/10.1111/1751-2980.12271

28. Nahar S, Mukhopadhyay AK, Khan R, et al. Antimicrobial susceptibility of Helicobacter pylori strains isolated in Bangladesh. J Clin Microbiol. 2004;42(10): 4856-4858. https://doi.org/10.1128/JCM.42.10.4856-4858.2004

29. Goodwin A, Kersulyte D, Sisson G, Veldhuyzen van Zanten SJ, Berg DE, Hoffman PS. Metronidazole resistance in Helicobacter pylori is due to null mutations in a gene (rdxA) that encodes an oxygen-insensitive NADPH nitroreductase. Mol Microbiol. 1998;28(2):383-393. https://doi.org/10.1046/j.1365-2958.1998.00806.x

30. Osato MS, Reddy R, Reddy SG, Penland RL, Malaty HM, Graham DY. Pattern of primary resistance of Helicobacter pylori to metronidazole or clarithromycin in the United States. Arch Intern Med. 2001;161(9):1217-1220. https://doi.org/ 10.1001/archinte.161.9.1217

31. Smith SI, Oyedeji KS, Arigbabu AO, Atimomo C, Coker AO. High amoxicillin resistance in Helicobacter pylori isolated from gastritis and peptic ulcer patients in Western Nigeria. J Gastroenterol. 2001;36(1):67-68. https://doi.org/10.1007/ s005350170158

32. Stella IS, Emmanuel AO, Helen AG, et al. Diagnostic methods for the detection of Helicobacter pylori in Nigeria. Trop Gastroenterol. 2010;31(2):113-115.

33. Hunt RH, Xiao SD, Megraud F, et al. Helicobacter pylori in developing countries. World Gastroenterology Organisation Global Guideline. J Gastrointestin Liver Dis. 2011;45(5):383-388.

34. Gisbert JP, Pajares JM. Stool antigen test for the diagnosis of Helicobacter pylori infection: A systematic review. Helicobacter. 2004;9(4):347-368. https://doi.org/ 10.1111/j.1083-4389.2004.00235.x 